International Journal of Pure and
SvedbergOpen
DISSEMNATIONoFkNowlLDGE
Research Paper

\title{
Simple Close Curve Magnetization and Application to Bellman's Lost in the Forest Problem
}

T. Agama ${ }^{1 *}$

'Department of Mathematics, African Institute for Mathematical Science, Ghana. E-mail: theophilus@aims.edu.gh/emperordagama@yahoo.com

\section{Article Info}

Volume 1, Issue 1, October 2021

Received : 06 January 2021

Accepted : 17 August 2021

Published : 05 October 2021

doi: 10.51483/IJPAMR.1.1.2021.48-54

\section{Abstract}

In this paper we introduce and develop the notion of simple close curve magnetization. We provide an application to Bellman's lost in the forest problem assuming special geometric conditions between the hiker and the boundary of the forest.

Keywords: Simple close curve, Magnetization, Magnetic field, Bellman, Forest, Hiker.

(C) 2021 T. Agama. This is an open access article under the CC BY license (https://creativecommons.org/licenses/by/4.0/), which permits unrestricted use, distribution, and reproduction in any medium, provided you give appropriate credit to the original author(s) and the source, provide a link to the Creative Commons license, and indicate if changes were made.

\section{Introduction and Problem Statement}

Bellman's lost in the forest problem is a central problem which lies at the interface of geometry and optimization. It has its origin dating back in 1995 by the American applied mathematician Bellman (1956). The problem has the following well-known formulation which can be found in Finch and Wetzel (2004).

Question 1 (Bellman's Lost in the Forest Problem): Given a hiker in the forest with his orientation within the forest unbeknownst to him, then what is the best possible decision to be taken to exit in the shortest possible time taking into consideration the shape of the forest and the dimension of the space covering the forest?

Much work has been done in studying this problem by a few authors (see Ward, 2008). This presumably has not to do with diminished interest and less popularity but may partly be attributed to the conceivable complexity of any viable tool to studying the problem. Regardless of its inherent difficulty a complete solution has been found and is known for a few class of shapes which are taken to be forest (Ward, 2008).

In this paper we introduce and develop the notion of magnetization of simple close curves. Using this notion we devise an algorithm that takes as input the shape of the forest and the dimension within which the forest resides and produce as output the optimal path to be taken by the hiker $\vec{v} \in \mathrm{R}^{n}$.

\section{Simple Close Curves with Magnetic Boundaries}

In this section we introduce the notion of simple close curve with magnetic boundaries equipped magnets. We study this concept and expose some connections with other notions.

\footnotetext{
* Corresponding author: T. Agama, Department of Mathematics, African Institute for Mathematical Science, Ghana. E-mail: theophilus@aims.edu.gh/emperordagama@yahoo.com
} 
Definition 2.1: Let $C$ be a simple close curve in $\mathrm{R}^{n}$ and $\mathrm{V}^{n}$ be the space of vectors in $\mathrm{R}^{n}$. Then by the magnetization of the interior of $C$, denoted $C_{I n t}$, with magnets $\vec{u}_{1}, \ldots, \vec{u}_{k}$ on the boundary $C_{B}$, we mean the map

$$
\Lambda_{\vec{u}_{1}, \vec{u}_{2}, \ldots, \vec{u}_{k}}: C_{\text {Int }} \rightarrow \mathrm{V}^{n}
$$

such that for any $\vec{v} \in C_{\text {Int }}$ with $\vec{v} \neq \vec{O}$ then

$$
\Lambda_{\vec{u}_{1}, \vec{u}_{2}, \ldots, \vec{u}_{k}}(\vec{v})=\Lambda_{\vec{u}_{j}}(\vec{v})=\vec{u}_{j}-\vec{v}
$$

with $\vec{v} \cdot \vec{u}_{j}=0$ if and only if $\left\|\vec{v}-\vec{u}_{j}\right\|=\min \left\{\left\|\vec{u}_{s}-\vec{v}\right\|\right\}_{s=1}^{k}$. We call

$$
\left\|\Lambda_{\vec{u}_{1}, \vec{u}_{2}, \ldots, \vec{u}_{k}}(\vec{v})\right\|=\left\|\Lambda_{\vec{u}_{j}}(\vec{v})\right\|=\left\|\vec{v}-\vec{u}_{j}\right\|
$$

the measure of magnetization. We denote the magnetic field of the magnet $\vec{u}_{j}$ by $O_{\vec{u}_{j}}$. Strictly speaking, we denote the $\varepsilon$-magnetic field of the magnet $\vec{u}_{j}$ by $O_{\vec{u}_{j}}(\varepsilon)$ and we say $\vec{v}_{i} \in O_{\vec{u}_{j}}(\varepsilon)$ if and only

$$
\left\|\vec{u}_{j}-\vec{v}_{i}\right\| \leq \varepsilon
$$

Definition 2.2: Let $C$ be a simple close curve in $\mathrm{R}^{n}$ with boundary $C_{B}$. Then we say the boundary is magnetic with magnets $\cup_{i=1}^{\infty}\left\{\vec{u}_{i}\right\} \in C_{B}$ if for any $\vec{u}_{j} \in C_{B}$ there exist some $\vec{u}_{i} \in \cup_{i=1}^{\infty}\left\{\vec{u}_{i}\right\}$ with

$$
\vec{u}_{i} \in O_{\vec{u}_{j}}(\varepsilon)
$$

for any $\varepsilon>0$ such that

$$
O_{\vec{u}_{i}}(\delta) \cap C_{I n t} \neq \phi
$$

and

$$
O_{\vec{u}_{i}}(\delta) \cap\left(\mathrm{R}^{n} \backslash C_{B} \cup C_{\text {Int }}\right) \neq \phi
$$

for any $\delta>0$. We denote with

$$
B\left(O_{\vec{u}_{i}}(\varepsilon)\right)
$$

the boundary of the neighbourhood $O_{\vec{u}_{i}}(\varepsilon)$.

The language as espoused in Definition 2.2 suggests very clearly that magnets are allowed to be dense on the boundary of their simple close $C$ with magnetic boundary $C_{B}$. This strict enforcement will ease the development of our geometry and related theories. In any case we can take the infinite set of magnets on the boundary of a typical simple close curve to be the entire boundary $C_{B} \subset \mathrm{R}^{n}$.

Remark 2.3: Next we put all simple close curves with magnetic boundaries equipped with magnets and their constant dilates into one single category. In essence we would consider two simple close curves with magnetic boundaries to be distinct if there exists a magnet on the boundary of one curve which fails to be a constant dilate on the other.

Proposition 2.1: Every simple close curve $C$ in $\mathrm{R}^{n}$ with magnetic boundary is uniquely determined by their magnetic boundary $C_{B}$ upto constant dilates of their magnets. 
Proof: Let $C$ be a simple closed curve with magnetic boundary $C_{B}$ in $\mathrm{R}^{n}$ endowed with magnets $\cup_{i=1}^{\infty}\left\{\vec{s}_{i}\right\}$ and $\cup_{i=1}^{\infty}\left\{\vec{t}_{i}\right\}$. Pick arbitrarily $\vec{v} \in C_{I n t}$ then applying the magnetization $\Lambda: C_{I n t} \rightarrow \mathrm{V}^{n}$ on the interior $C_{I n t}$, we have the equality

$$
\Lambda_{\cup_{i=1}^{\infty}\left\{\vec{s}_{i}\right\}}(\vec{v})=\Lambda_{\vec{s}_{j}}(\vec{v})
$$

for some $1 \leq j$ with $\vec{v} \cdot \vec{s}_{j}=0$ if and only if $\left\|\vec{v}-\vec{s}_{j}\right\|=\min \left\{\left\|\vec{s}_{i}-\vec{v}\right\|\right\}_{i=1}^{\infty}$ and

$$
\Lambda_{\cup_{i=1}^{\infty}\left\{\vec{t}_{i}\right\}}(\vec{v})=\Lambda_{\vec{t}_{k}}(\vec{v})
$$

for some $1 \leq k$ with $\vec{t}_{k} \cdot \vec{v}=0$ if and only if $\left\|\vec{v}-\vec{t}_{k}\right\|=\min \left\{\left\|\vec{t}_{i}-\vec{v}\right\|\right\}_{i=1}^{\infty}$. It follows that $\lambda \vec{s}_{j}=\vec{t}_{k}$ for $\lambda \in \mathrm{R}$. Since the vector $\vec{v}$ is an arbitrary point in $C_{I n t}$ the claim follows immediately.

Theorem 2.4: Let $C$ be a simple closed curve in $\mathrm{R}^{n}$ with magnetic boundary $C_{B}$ equipped with magnets $\cup_{i=1}^{\infty}\left\{\vec{u}_{i}\right\}$. For any $\vec{v} \in C_{I n t}$ such that $\vec{v} \neq \vec{O}$ then $\Lambda_{\cup_{i=1}^{\infty}\left\{\vec{u}_{i}\right\}}(\vec{v})=\Lambda_{\vec{u}_{j}}(\vec{v})_{\text {with }} \vec{v} \cdot \vec{u}_{j}=0$ if and only if there exists $\varepsilon>0$ such that $\vec{u}_{j} \in O_{\vec{v}}(\varepsilon)$ and

$$
\vec{u}_{s} \notin O_{\vec{v}}(\varepsilon)
$$

for all $s \neq j$.

Proof: First let $C$ be a simple closed curve in $\mathrm{R}^{n}$ with magnetic boundary $C_{B}$ equipped with magnets $\cup_{i=1}^{\infty}\left\{\vec{u}_{i}\right\}$. Let us pick arbitrarily $\vec{v} \in C_{I n t}$ such that $\vec{v} \neq \vec{O}$, apply the magnetization $\Lambda: C_{I n t} \rightarrow \mathrm{V}^{n}$ and suppose

$$
\Lambda_{\cup_{i=1}^{\infty}\left\{\vec{u}_{i}\right\}}(\vec{v})=\Lambda_{\vec{u}_{j}}(\vec{v})
$$

with $\vec{v} \cdot \vec{u}_{j}=0$, then $\left\|\vec{v}-\vec{u}_{j}\right\|=\min \left\{\left\|\vec{u}_{s}-\vec{v}\right\|\right\}_{s=1}^{\infty}$ for all $\vec{u}_{s} \in \cup_{i=1}^{\infty}\left\{\vec{u}_{i}\right\}$. Now choose $\varepsilon=\left\|\vec{u}_{j}-\vec{v}\right\|$ and construct the neighbourhood $O_{\vec{v}}(\varepsilon)$. By virtue of the construction $\vec{u}_{j} \in O_{\vec{v}}(\varepsilon)$. We claim that $\vec{u}_{s} \notin O_{\vec{v}}(\varepsilon)$ for all $s \neq j$ with

$$
\vec{u}_{s} \in \cup_{i=1}^{\infty}\left\{\vec{u}_{i}\right\}
$$

Let us suppose to the contrary that there exist at least some $\vec{u}_{t} \in \cup_{i=1}^{\infty}\left\{\vec{u}_{i}\right\} \subset C_{B}$ with $t \neq j$ such that $\vec{u}_{t} \in O_{\vec{v}}(\varepsilon)$. Then there exist some $\vec{u}_{k} \in \cup_{i=1}^{\infty}\left\{\vec{u}_{i}\right\} \subset C_{B}$ with $k \neq j$ such that $\vec{u}_{k} \in B\left(O_{\vec{v}}(\varepsilon)\right) \cap C_{B}$ so that

$$
\left\|\vec{v}-\vec{u}_{k}\right\|=\varepsilon=\min \left\{\left\|\vec{u}_{s}-\vec{v}\right\|\right\}_{s=1}^{\infty}
$$

Applying the magnetization, It follows that

$$
\Lambda_{\cup_{i=1}^{\infty}\left\{\vec{u}_{i}\right\}}(\vec{v})=\Lambda_{\vec{u}_{k}}(\vec{v})
$$


with $\vec{v} \cdot \vec{u}_{k}=0$. The upshot is that $\vec{u}_{j}=\lambda \vec{u}_{k}$ for $\lambda \neq 1$ so that

$$
\varepsilon=\left\|\vec{v}-\vec{u}_{k}\right\| \neq\left\|\vec{v}-\vec{u}_{j}\right\|
$$

which contradicts our choice of $\left\|\vec{v}-\vec{u}_{j}\right\|=\varepsilon>0$.

Conversely suppose there exists $\varepsilon>0$ such that $\vec{u}_{j} \in O_{\vec{v}}(\varepsilon)$ and

$$
\vec{u}_{s} \notin O_{\vec{v}}(\varepsilon)
$$

for all $s \neq j$. Then

$$
\left\|\vec{v}-\vec{u}_{j}\right\|=\min \left\{\left\|\vec{u}_{s}-\vec{v}\right\|\right\}_{s=1}^{\infty}
$$

for all $\vec{u}_{s} \in \cup_{i=1}^{\infty}\left\{\vec{u}_{i}\right\}$ and $\vec{v} \in C_{\text {Int }}$. The claim follows immediately from this assertion by applying the magnetization $\Lambda: C_{I n t} \rightarrow \mathrm{V}^{n}$.

Theorem 2.5: Let $C$ be a simple close curve in $\mathrm{R}^{n}$ with magnetic boundary $C_{B}$ equipped with magnets $\cup_{i=1}^{\infty}\left\{\vec{u}_{i}\right\}$. Then for any $\vec{v} \in C_{\text {Int }}$ such that $\vec{v} \neq \vec{O}$ there exists some $\varepsilon>0$ such that $\vec{u}_{j} \in O_{\vec{v}}(\varepsilon)$ for some $\vec{u}_{j} \in \cup_{i=1}^{\infty}\left\{\vec{u}_{i}\right\}$ and

$$
\vec{u}_{t} \notin O_{\vec{v}}(\varepsilon)
$$

with $t \neq j$ for all $\vec{u}_{t} \in \cup_{i=1}^{\infty}\left\{\vec{u}_{i}\right\}$

Proof: Let $C_{B}$ and $C_{I n t}$ be the magnetic boundary and the interior of the simple close curve $C$, respectively. Let $\cup_{i=1}^{\infty}\left\{\vec{u}_{i}\right\} \subset C_{B}$ be the magnets on the boundary. Pick arbitrarily $\vec{v} \in C_{I n t}$ such that $\vec{v} \neq \vec{O}$, then for each $\vec{u}_{j} \in \cup_{i=1}^{\infty}\left\{\vec{u}_{i}\right\}$ there exists $\varepsilon>0$ such that

$$
\vec{u}_{j} \in O_{\vec{v}}(\varepsilon)
$$

Let us choose

$$
O_{\vec{v}}(\delta)=\min \left\{O_{\vec{v}}(\varepsilon) \mid \vec{u}_{j} \in O_{\vec{v}}(\varepsilon), \varepsilon>0\right\}
$$

for each $\vec{u}_{j} \in \cup_{i=1}^{\infty}\left\{\vec{u}_{i}\right\}$. Let $\vec{u}_{j} \in O_{\vec{v}}(\delta)$ then it follows that $\left\|\vec{u}_{j}-\vec{v}\right\|=\delta$ by virtue of (4.1) and

$$
\left\|\vec{u}_{j}-\vec{v}\right\|=\delta=\min \left\{\left\|\vec{v}-\vec{u}_{s}\right\|: \vec{u}_{s} \in \cup_{i=1}^{\infty}\left\{\vec{u}_{i}\right\}\right\}
$$

Apply the magnetization $\Lambda: C_{I n t} \rightarrow \mathrm{V}^{n}$, then we have

$$
\Lambda_{\cup_{i=1}^{\infty}\left\{\vec{u}_{i}\right\}}(\vec{v})=\Lambda_{\vec{u}_{j}}(\vec{v})
$$

with $\vec{u}_{j} \cdot \vec{v}=0$. Let us suppose to the contrary $\vec{u}_{s} \in O_{\vec{v}}(\delta)$ for at least some $\vec{u}_{s} \in \cup_{i=1}^{\infty}\left\{\vec{u}_{i}\right\}$ with $j \neq s$. Then it follows that there exists some $\vec{u}_{t} \in \cup_{i=1}^{\infty}\left\{\vec{u}_{i}\right\}$ with $t \neq j$ such that 
$\vec{u}_{t} \in C_{B} \cap B\left(O_{\vec{v}}(\delta)\right)$

It follows that

$$
\left\|\vec{v}-\vec{u}_{t}\right\|=\delta=\min \left\{\left\|\vec{v}-\vec{u}_{s}\right\|: \vec{u}_{s} \in \cup_{i=1}^{\infty}\left\{\vec{u}_{i}\right\}\right\}
$$

so that by applying the magnetization $\Lambda: C_{I n t} \rightarrow \mathrm{V}^{n}$, we have

$$
\Lambda_{\cup_{i=1}^{\infty}\left\{\vec{u}_{i}\right\}}(\vec{v})=\Lambda_{\vec{u}_{t}}(\vec{v})
$$

with $\vec{u}_{t} \cdot \vec{v}=0$. It follows that there exists some $\alpha \neq 1$ such that $\vec{u}_{t}=\alpha \vec{u}_{j}$ so that

$$
\left\|\vec{v}-\vec{u}_{t}\right\|=\delta \neq\left\|\vec{v}-\vec{u}_{j}\right\|
$$

thereby contradicting (2.2). This completes the proof of the theorem.

\section{Connected and Isomorphic Simple Close Curves with Magnetic Boundaries}

In this section we introduce a classification scheme for all simple close curves $C$ in $\mathrm{R}^{n}$ with magnetic boundaries $C_{B}$. This scheme pretty much allows us to put all similar such simple close curves into a single family and choose a representative for our work.

Definition 3.1: Let $C_{1}$ and $C_{2}$ be simple close curves with magnetic boundaries equipped with magnets $\cup_{i=1}^{\infty}\left\{\vec{t}_{i}\right\}$ and $\cup_{i=1}^{\infty}\left\{\vec{w}_{i}\right\}$, respectively. Then we say $C_{1}$ and $C_{2}$ are connected if there exists some $\vec{t}_{j} \in \cup_{i=1}^{\infty}\left\{\vec{t}_{i}\right\}$ and some $\vec{w}_{k} \in \cup_{i=1}^{\infty}\left\{\vec{w}_{i}\right\}$ such that

$$
\vec{t}_{j}=\lambda \vec{w}_{k}
$$

for some $\lambda \in \mathrm{R}$. We denote the connection by $C_{1} \rightleftharpoons C_{2}$. We say $C_{1}$ and $C_{2}$ are isomorphic if the connection exists for each $\vec{t}_{j} \in \cup_{i=1}^{\infty}\left\{\vec{t}_{i}\right\}$ and $\vec{w}_{k} \in \cup_{i=1}^{\infty}\left\{\vec{w}_{i}\right\}$.

We denote the isomorphism by $C_{1} \smile C_{2}$.

Proposition 3.1: Let $C_{1}$ and $C_{2}$ be simple close curves with magnetic boundaries equipped with magnets $\cup_{i=1}^{\infty}\left\{\vec{t}_{i}\right\}$ and $\cup_{i=1}^{\infty}\left\{\vec{w}_{i}\right\}$, respectively. If $C_{1} \subset C_{2}$ then $C_{1} \bigcup C_{2}$.

Proof: Suppose $C_{1} \subset C_{2}$ and let $C_{1 n t}$ and $C_{2 l n t}$ be their interior, respectively. Next pick arbitrarily a point $\vec{v} \in C_{1 I n t}$ such that $\vec{v} \neq \vec{O}$, then $\vec{v} \in C_{2 \text { Int }}$ so that

$$
\min \left\{\left\|\vec{t}_{s}-\vec{v}\right\|\right\}_{s=1}^{\infty} \leq \min \left\{\left\|\vec{w}_{s}-\vec{v}\right\|\right\}_{s=1}^{\infty}
$$

Let us set

$$
\left\|\vec{t}_{j}-\vec{v}\right\|=\min \left\{\left\|\vec{t}_{s}-\vec{v}\right\|: \vec{t}_{s} \in \cup_{i=1}^{\infty}\left\{\vec{t}_{i}\right\}\right\}_{s=1}^{\infty}
$$


and

$$
\left\|\vec{w}_{k}-\vec{v}\right\|=\min \left\{\left\|\vec{w}_{s}-\vec{v}\right\|: \vec{w}_{s} \in \cup_{i=1}^{\infty}\left\{\vec{w}_{i}\right\}\right\}_{s=1}^{\infty}
$$

and apply the magnetization $\Lambda: C_{1 \text { Int }} \rightarrow \mathrm{V}^{n}$ and $\Lambda: C_{2 \text { Int }} \rightarrow \mathrm{V}^{n}$, then we obtain the following paths

$$
\Lambda_{\cup_{i=1}^{\infty}\left\{\vec{f}_{i}\right\}}(\vec{v})=\Lambda_{\vec{t}_{j}}(\vec{v})=\vec{t}_{j}-\vec{v}
$$

with $\vec{t}_{j} \cdot \vec{v}=0$ and

$$
\Lambda_{\cup_{i=1}^{\infty}\left\{\vec{w}_{i}\right\}}(\vec{v})=\Lambda_{\vec{w}_{k}}(\vec{v})=\vec{w}_{k}-\vec{v}
$$

with $\vec{w}_{k} \cdot \vec{v}=0$. It follows that $\vec{w}_{k}=\lambda \vec{t}_{j}$ for some $\lambda \in \mathrm{R}$. Since $\vec{v}$ was taken arbitrarily and for any such choice there is a unique choice of magnet on the boundary of each simple close curve minimizing the distance from $\vec{v}$, the claim follows.

\section{Application to Bellman's Lost in the Forest Problem}

This section pretty much illustrates a sketch of an application of the underlying notion to Bellman's lost in the forest problem. The solution is quite algorithmic in nature but works primarily in parallel with the above developments. The Bellman lost in the forest problem is one of the most important problems in the area of optimization, yet we find the following tools developed in the foregone section useful. The problem is often stated in the following manner:

Question 2 (Bellman's Lost in the Forest Problem): Given a hiker lost in the forest with his orientation unknown, what is the best decision to be taken to exit in the shortest possible time taking into into consideration the shape of the boundary of the forest and the dimension of the space covering the forest?

\subsection{A Sketch Partial Solution}

First we classify the infinite collection of simple close curves in $\mathrm{R}^{n}$ with magnetic boundaries according as they are isomorphic.

That is, we consider the partition

$$
\mathrm{M}:=\bigcup_{k=1}^{\infty}\left\{C_{i} \subset \mathrm{R}^{n} \mid C_{i} \bigcup^{\cup} C_{k}\right\}
$$

such that $\left\{C_{i} \subset \mathrm{R}^{n} \mid C_{i} \cup^{\cup} C_{k}\right\} \cap\left\{C_{i} \subset \mathrm{R}^{n} \mid C_{i} \cup^{\cup} C_{j}\right\}=\phi$ for $k \neq j$ and $C_{k}$ and $C_{j}$ are not isomorphic. Let us pick arbitrarily a simple close curve $C$ arbitrarily in $\mathrm{M}$.

Then it follows that

$$
C \in\left\{C_{i} \subset \mathrm{R}^{n} \mid C_{i} \cup_{C_{k}}\right\}
$$

for some $k \geq 1$. The simple close curve $C$ with magnetic boundary equipped with magnets $\cup_{i=1}^{\infty}\left\{\vec{u}_{i}\right\}$ is uniquely determined upto constant dilates of magnets by virtue of Proposition 2.1. Now let $\vec{v} \neq \vec{O}$ be an arbitrary point (hiker) lost in $C_{I n t}$ (forest).

Appealing to Theorem 2.5 we choose 


$$
O_{\vec{v}}(\delta)=\min \left\{O_{\vec{v}}(\varepsilon) \mid \vec{u}_{j} \in O_{\vec{v}}(\varepsilon), \varepsilon>0\right\}
$$

for each $\vec{u}_{j} \in \cup_{i=1}^{\infty}\left\{\vec{u}_{i}\right\}$. By virtue of our choice there exists some magnet $\vec{u}_{t} \in O_{\vec{v}}(\delta)$ for $\vec{u}_{t} \in \cup_{i=1}^{\infty}\left\{\vec{u}_{i}\right\}$ since magnets are dense on the boundary $C_{B}$ by virtue of Definition 2.2. Next we apply the magnetization $\Lambda: C_{I n t} \rightarrow \mathrm{V}^{n}$ and obtain the exit path

$$
\Lambda_{\cup_{i=1}^{\infty}\left\{\vec{u}_{i}\right\}}(\vec{v})=\Lambda_{\vec{u}_{t}}(\vec{v})=\vec{u}_{t}-\vec{v}
$$

with $\vec{v} \cdot \vec{u}_{t}=0$ by appealing to Theorem 2.4 with the least measure of magnetization

$$
\left\|\vec{v}-\vec{u}_{t}\right\|=\min \left\{\left\|\vec{v}-\vec{u}_{s}\right\|: \vec{u}_{s} \in \cup_{i=1}^{\infty}\left\{\vec{u}_{i}\right\}\right\}
$$

Thus the hiker exits the forest without being privy to information of his orientation within the forest with the shortest path $\vec{u}_{t}-\vec{v}$.

It has to be said that the solution as espoused in the sketch may not be viewed as a complete solution to the Bellman lost in the forest problem, because in practice the equivalence of the minimal distance of the hiker $\vec{v}$ to the boundary of the simple close curve (forest) $C_{B}$ with the notion magnetization $\Lambda: C_{I n t} \rightarrow \mathrm{V}^{n}$ under the additional orthogonality condition of the hiker and some point on the boundary of the forest $\vec{v} \cdot \vec{u}_{t}=0$ may not necessarily hold. Nonetheless we believe it is still possible the problem could be studied under the equivalence.

$$
\Lambda_{\vec{u}_{1}, \vec{u}_{2}, \ldots, \vec{u}_{k}}(\vec{v})=\Lambda_{\vec{u}_{j}}(\vec{v})=\vec{u}_{j}-\vec{v}
$$

if and only if $\left\|\vec{v}-\vec{u}_{j}\right\|=\min \left\{\left\|\vec{u}_{s}-\vec{v}\right\|\right\}_{s=1}^{k}$ without the extra regime $\vec{v} \cdot \vec{u}_{j}=0$ for any hiker in the forest.

\section{References}

Bellman, Richard. (1956). Minimization Problem. Bull. Amer. Math. Soc., 62(3), 270.

Finch, Steven, R., and Wetzel, John, E. (2004). Lost in a Forest The American Mathematical Monthly, 111(8), 645-654. Taylor \& Francis.

Ward, John, W, (2008). Exploring the Bellman Forest Problem. 